\title{
Cervical osteogenic degeneration in Japanese professional wrestlers and its relationship to cervical spine injury
}

\author{
Manabu Sasaki, MD, PhD, ${ }^{1}$ Shunji Asamoto, MD, PhD, ${ }^{2}$ Masao Umegaki, MD, PhD, ${ }^{1}$ and \\ Katsumi Matsumoto, MD, PhD1
'Department of Neurosurgery and Spine Surgery, Iseikai Hospital, Osaka; and 'Department of Neurosurgery, Makita General Hospital, Tokyo, Japan

\begin{abstract}
OBJECTIVE This study aimed to investigate the characteristics of cervical degeneration in Japanese professional wrestlers and its relationship with the risk of cervical spine injury (CSI).

METHODS Since 2012, 27 Japanese male wrestlers belonging to a professional wrestling association have undergone periodical medical examinations of the cervical spine. If neurological symptoms were observed in the wrestlers, the resident trainers urged them to undergo a brief examination at the authors' institutions. In addition to this prospective research study, the mechanisms of the CSIs that occurred in 5 wrestlers, including 2 with CSI before 2012 and 3 who were independent from the professional wrestling association, were retrospectively investigated by reviewing the circumstances of the injury and the wrestlers' imaging studies.
\end{abstract}

RESULTS The mean age of the wrestlers was 36.9 years (range $23-56$ years) at the initial examination. An anterior giant ossifying lesion (AGOL) was observed in the anterior aspect of the cervical spine of 11 wrestlers (41\%). The AGOLs tended to grow and spread to multiple spinal levels as the wrestlers aged. Of the 12 wrestlers with osteogenic lesions, 10 older than 40 years of age (83\%) had an AGOL, which is frequently accompanied by osseous spinal canal stenosis. Two wrestlers presented with spinal cord compression with intramedullary intensity change on MRI. However, during the follow-up period, no spinal cord injury (SCl) occurred in the wrestlers, although thoracolumbar injury occurred in 2 wrestlers during a match. In examining the 5 wrestlers with CSI, the injury occurred at the spinal levels without an AGOL. The most frequent pathology of CSI (60\%) was SCI at the spinal level adjacent to the multilevel AGOL.

CONCLUSIONS AGOL is a peculiar cervical degeneration of Japanese professional wrestlers, especially in aged wrestlers. The AGOL appears to be a biological reaction to reinforce the anterior aspect of the cervical spine of professional wrestlers, who routinely defend themselves in a flexed neck posture against their opponent. The present results suggest that the risk of CSI is not increased by spinal canal stenosis accompanied by AGOL. Further studies are needed to investigate the relationship between the wrestlers' cervical degeneration and the risk of CSI in more detail.

https://thejns.org/doi/abs/10.3171/2018.5.SPINE18283

KEYWORDS professional wrestlers; ossification of the anterior longitudinal ligament; giant osteophyte; osteogenic degeneration; cervical spine injury; spinal cord injury

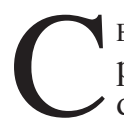

ERVICAL spine injury (CSI) commonly occurs in participants of collision sports. However, the condition of the cervical spine is rarely known before the injury. In 2009, a famous Japanese professional wrestler went into cardiopulmonary arrest just after he received a throwing move called a "back suplex," in which he was thrown backward by his opponent and dropped flat on his back. The fatal condition was induced by severe spinal cord injury (SCI) at the high cervical spine. Thereafter, a potential risk of CSI was recognized by the wrestlers and persons concerned with professional wrestling. In 2012, a major Japanese professional wrestling association requested a periodical medical examination (PME) of the cervical spine; thereafter, we have been performing PMEs of the wrestlers who belong to the association. After we started performing PMEs, some wrestlers with CSI who

ABBREVIATIONS AGOL = anterior giant ossifying lesion; $C S I=$ cervical spine injury; $\mathrm{OALL}=$ ossification of the anterior longitudinal ligament; $\mathrm{OPLL}=0 \mathrm{Ossification}$ of the posterior longitudinal ligament; $\mathrm{PME}=$ periodical medical examination; $\mathrm{SCl}=$ spinal cord injury; $\mathrm{VA}=$ vertebral assimilation .

SUBMITTED March 7, 2018. ACCEPTED May 25, 2018.

INCLUDE WHEN CITING Published online September 21, 2018; DOI: 10.3171/2018.5.SPINE18283. 
were independent from the association were also treated. These experiences enabled us to follow the natural course of their cervical spine degeneration and understand its relationship with CSI. In this study, we investigated the peculiar osteogenic degeneration at the anterior aspect of the wrestlers' cervical spine and its relationship with CSI.

\section{Methods}

\section{Prospective Study on the Wrestlers' Cervical Degeneration and Its Relationship With CSI}

In 2012, one of the major professional wrestling associations in Japan requested that we perform PMEs of the wrestlers' cervical spines to reduce the risk of CSI. We accepted the request and decided to establish a medical committee within the association to research the wrestlers' cervical degeneration and determine some measures to reduce the risk of CSI. All 27 wrestlers belonging to the association were required to undergo a physical examination and imaging studies annually. The wrestlers knew that the data would be used in our study and project for the prevention of CSI, and informed consent was given at the medical examination. The imaging studies included dynamic plain radiography, MRI, and CT. Radiological assessment was performed by 3 authors (M.S., S.A., and M.U.) who are board-certified spine surgeons of the Neurospinal Society of Japan. If there was disagreement in the assessment between the authors, the final decision was made by the majority of authors. The anteroposterior diameter of the spinal canal was measured at the narrowest part of the spinal canal on the midsagittal plane of the CT scan. Spinal canal stenosis was noted when the diameter was smaller than $12 \mathrm{~mm}$. The stenosis was considered severe when the diameter was smaller than $10 \mathrm{~mm}$. The condition of the cervical spine was explained to each wrestler, and they were informed of the potential risk of CSI with moderate or severe degeneration. The wrestlers who presented with spinal cord compression with intramedullary signal change on MRI were recommended to retire from their careers. However, if they desired to continue their careers, we accepted their decision because no criteria for retirement were established. The resident trainers continuously monitored the wrestlers' body condition. When a wrestler presented with neurological symptoms or abnormal findings, he was urged to undergo a brief medical examination at our institutions.

\section{Retrospective Study of CSI Occurring in the Wrestlers}

Two wrestlers belonging to the association sustained CSI during their wrestling matches before the PMEs started. Our institution (Iseikai Hospital) was also requested to treat 3 wrestlers independent from the association who sustained CSI during their matches since 2012. The mechanism of CSI in these 5 wrestlers was retrospectively examined by reviewing the circumstance of the injuries and their imaging studies.

Informed consent and permission for publication were obtained from each participant of this retrospective study. The study protocol was approved by the ethics committee of Iseikai Hospital.

\section{Results \\ Prospective Study on Cervical Degeneration in the Wrestling Association}

Table 1 shows a summary of the initial examination of the 27 wrestlers. None of the wrestlers dropped out from the PME. The wrestlers' mean age was 36.9 years (range $23-56$ years) at the initial examination.

An osteogenic lesion was observed in 12 wrestlers; an anterior giant ossifying lesion (AGOL) (Fig. 1), a large osseous lesion formed at the anterior aspect of the cervical spine, in 11 wrestlers (41\%); vertebral assimilation (VA) in 5 wrestlers $(18 \%)$; and ossification of the posterior longitudinal ligament (OPLL) in 3 wrestlers (11\%). An AGOL was frequent in wrestlers over 40 years of age (73\%), whereas it was observed in only $19 \%(3 / 16)$ of the younger wrestlers. The AGOLs usually grew (Fig. 1C and F) and spread to multiple spinal levels as the wrestlers aged. VA was observed in 5 wrestlers more than 40 years old who also had an AGOL. Spinal canal stenosis was observed in 16 of 27 wrestlers (59\%; Table 1). Severe stenosis was present in 5 wrestlers, and 4 of them were more than 40 years old. Spinal canal stenosis was observed in 9 of 11 wrestlers with an AGOL (82\%), and the stenosis was severe in 3 wrestlers (27\%) more than 40 years old. In 8 of 11 wrestlers with an AGOL (73\%), the narrowest part of the spinal canal was present at the spinal level of AGOL. Spinal cord compression with intramedullary signal intensity change on MRI was observed in 2 wrestlers, but they lacked neurological symptoms or remarkable abnormality on the neurological examination. They were informed of the anticipation that the degeneration would increase the risk of SCI during their performance. However, both wrestlers determined to continue their activities as professional wrestlers.

During the follow-up period between 2012 and 2017, CSI did not occur in any of the wrestlers, but thoracolumbar spine injuries occurred in 2 wrestlers: vertebral fracture and epidural hematoma at the T12 level in case 4, and avulsion of the left L3 nerve root in case 13 .

\section{Retrospective Study of the 5 Wrestlers Who Had CSI}

Table 2 shows a summary of the 5 wrestlers who sustained CSI during their wrestling matches. The 2 wrestlers belonging to the association (cases 19 and 24) had CSI before the PMEs started in 2012. The other 2 (cases 28 and 29) sustained CSI in the wrestling matches held by the association after 2012. However, they had not undergone PME because they did not have a contract for regular employment with the association. The fifth wrestler (case 30) had previously established a temporary contract with the association. He sustained CSI during a wrestling match while with the other association after 2012. He asked us to provide treatment because the other association had no system for medical support. An AGOL with or without VA was observed in the 3 wrestlers more than 40 years of age (cases 24, 29, and 30; Fig. 2), and OPLL was present in 2 (cases 19 and 29). Spinal canal stenosis was noted in the 2 wrestlers with an AGOL (cases 29 and 30). In case 29, stenosis was noted at the spinal level with AGOL, but the narrowest part was present at the C3-4 levels with OPLL. 
TABLE 1. Summary of the initial examination of the 27 professional wrestlers belonging to a professional wrestling association

\begin{tabular}{|c|c|c|c|c|c|c|c|c|}
\hline $\begin{array}{l}\text { Case } \\
\text { No. }\end{array}$ & $\begin{array}{l}\text { Age } \\
\text { (yrs) }\end{array}$ & $\begin{array}{l}\text { Spinal Level } \\
\text { of AGOL }\end{array}$ & $\begin{array}{c}\text { Spinal } \\
\text { Level of VA }\end{array}$ & $\begin{array}{l}\text { Spinal Level } \\
\text { of OPLL }\end{array}$ & SCS & $\begin{array}{c}\text { SCS at Level } \\
\text { w/ AGOL }\end{array}$ & SCC & Spinal Injury During Wrestling Match \\
\hline 1 & 23 & & & & & & & \\
\hline 2 & 26 & & & & & & & \\
\hline 3 & 26 & & & & & & & \\
\hline 4 & 29 & & & & + & & & Vertebral fracture, epidural hematoma at T12 level in 2014 \\
\hline 5 & 30 & & & & + & & & \\
\hline 6 & 30 & & & & + & & & \\
\hline 7 & 31 & & & & & & & \\
\hline 8 & 31 & C4-5 & & & + & + & & \\
\hline 9 & 31 & & & & & & & \\
\hline 10 & 33 & C2-3 & & & + & & & \\
\hline 11 & 33 & & & & + & & & \\
\hline 12 & 34 & & & & & & & \\
\hline 13 & 34 & & & & + & & & Avulsion of It L3 nerve root in 2013 \\
\hline 14 & 35 & & & & & & & \\
\hline 15 & 36 & & & & + & & & \\
\hline 16 & 38 & C4-5 & & C5-6 & ++ & + & + & \\
\hline 17 & 40 & & & & & & & \\
\hline 18 & 41 & C5-7 & & & + & + & & \\
\hline 19 & 42 & & & C3-4 & + & & & C3-4 CSI in 2009 \\
\hline 20 & 43 & $\mathrm{C} 2, \mathrm{C} 3, \mathrm{C} 6, \mathrm{C} 7$ & C2-3 & & + & + & & \\
\hline 21 & 44 & C3-6 & & & ++ & ++ & & \\
\hline 22 & 44 & C3-4 & C5-7 & & ++ & + & & \\
\hline 23 & 45 & C4-6 & C5-6 & C5-6 & ++ & ++ & + & \\
\hline 24 & 45 & C3-7 & C3-7 & & & & & C3-4 CSI in 2011 \\
\hline 25 & 47 & C4-5 & C3-4, C5-6 & & ++ & ++ & & \\
\hline 26 & 48 & & & & & & & \\
\hline 27 & 56 & C6-7 & & & & & & \\
\hline
\end{tabular}

SCC = spinal cord compression with intramedullary signal intensity change on MRI; SCS = spinal canal stenosis $(+=$ the anteroposterior diameter of the spinal canal was $10-12 \mathrm{~mm} ;++=$ the anteroposterior diameter was $<10 \mathrm{~mm}$ ).

SCI at the C3-4 levels with OPLL but without an AGOL occurred in 1 wrestler (case 19) when he received the opponent's finishing move in which his body was dropped flat on the back in an extended neck posture with his head fixed on the opponent's knee. The other 4 wrestlers sustained CSI when they defended themselves in a flexed neck posture against their opponent. One wrestler (case 28), who lacked cervical degeneration, had a hangman's fracture, and the other 3 (cases 24, 29, and 30) had SCI without bone injury at the C3-4 levels (Fig. 2). MRI of the latter wrestlers showed signal intensity change of the spinal cord at the C3-4 levels, which was adjacent to the AGOL.

\section{Discussion}

The present study describes the peculiar osteogenic degeneration at the anterior aspect of the vertebral body of Japanese professional wrestlers. Some previous studies have reported such giant osseous lesions of ordinary persons that caused dysphagia. They described the lesions as giant osteophytes, ${ }^{5,11}$ ossification of the anterior longitudi- nal ligament (OALL), ${ }^{6,12}$ and/or diffuse idiopathic skeletal hyperostosis. ${ }^{7,9}$ In this study we describe the giant osseous lesion as an AGOL because we could not confirm whether the origin of the lesion was the bone or ligament. The present data showed that prevalence rates of AGOL and OPLL were $41 \%$ and $11 \%$, respectively, in 27 wrestlers with a mean age of 36.9 years; previous studies reported that the prevalence rates of OALL and OPLL were $11 \%$ and $8.3 \%$, respectively, in healthy Japanese men with a mean age of $56 \pm 12$ years. ${ }^{4}$ The prevalence of OPLL, which is generated by genetics, was almost identical between the wrestlers and ordinary persons, whereas that of AGOL is markedly higher in wrestlers than in ordinary persons. This result suggests that an AGOL is caused by the wrestlers' activity, not by genetics.

An AGOL appears to be a biological reaction to the external loading on the cervical spine during professional wrestlers' performance. Wrestlers routinely defend themselves against their opponent in a flexed neck posture, which reduces the risk of SCI triggered by hyperextension of the neck. As reported previously, long-term and 


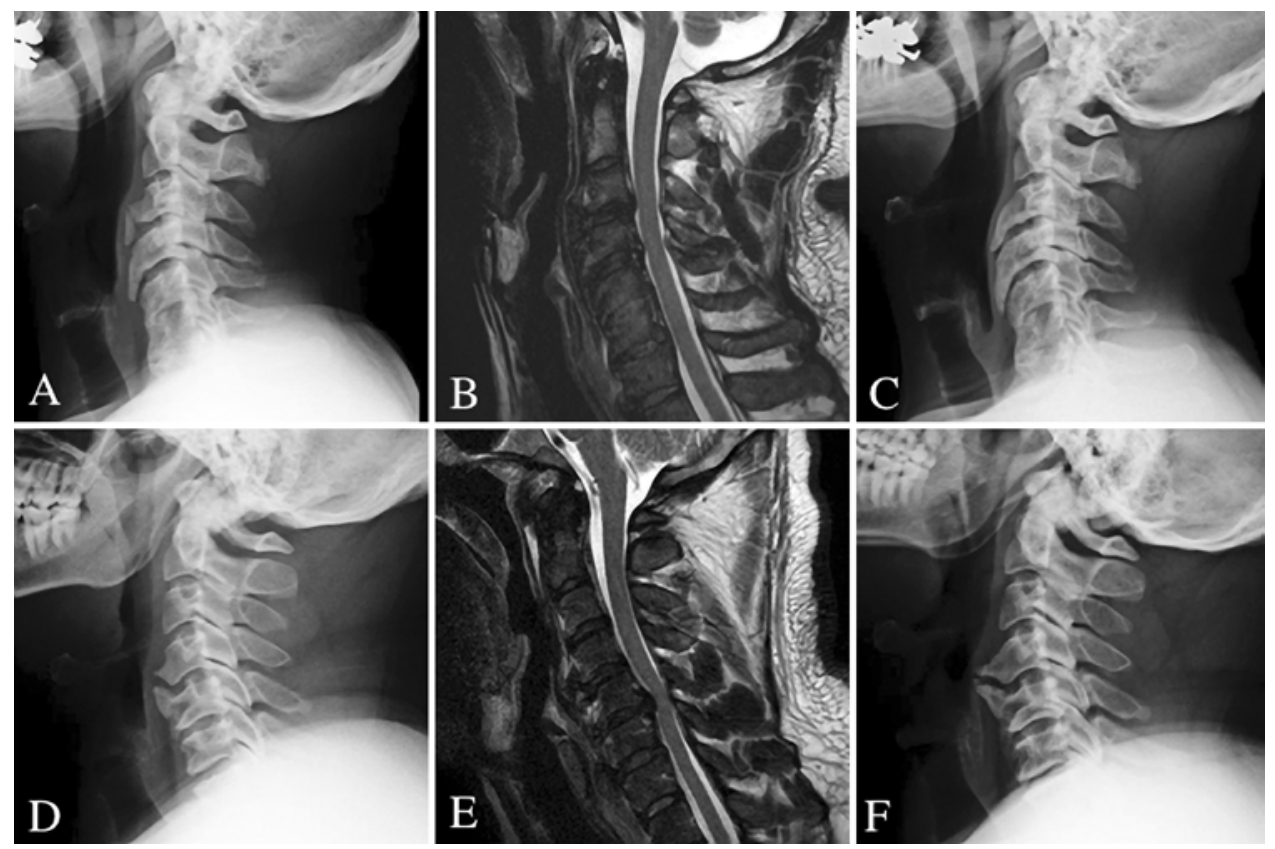

FIG. 1. Imaging studies of a 44-year-old wrestler (A-C, case 22) and a 38-year-old wrestler (D-F, case 16). The initial plain radiograph (A) and MR image (B) of the former wrestler show an AGOL at the C3-4 levels, VA at the C5-7 levels, and spinal canal stenosis at the C3-5 levels. The initial plain radiograph (D) and MR image (E) of the latter wrestler show an AGOL at the C4-5 levels, OPLL at the C5-6 levels, and spinal cord compression with intramedullary signal intensity change. The plain radiographs taken 5 years later ( $\mathbf{C}$ and $\mathbf{F}$ ) show that the AGOL of both wrestlers increased in size.

repetitive impact loading on the bone stimulates an osteogenic reaction, which produces adequate cortical bone and suitable distribution against the loading..$^{1,3,8}$ It can be considered that the AGOL was generated at the anterior part of the cervical spine against the repetitive impact loading during the defensive move in the flexed neck posture. Players of other collision sports, such as rugby football and American football, also use the flexed neck position to defend themselves against their opponent. To the best of our knowledge, no report is available on the development of AGOLs in athletes. Players of other collision sports rarely continue their careers over 40 years of age, which is the age at which generation of AGOLs in professional wrestlers accelerates. This may be one of the reasons for the lack of reports on AGOLs in sports players.

The present study suggests that cervical degeneration including AGOLs does not increase the risk of CSI. As shown in Table 1, AGOL is frequently accompanied by spinal canal stenosis. We anticipated that the stenosis would induce SCI during the wrestlers' performance. Although the wrestlers had such strenuous matches that they caused thoracolumbar injuries in 2 cases, none of the resident wrestlers had SCI during the follow-up period from 2012 to 2017. In addition, the retrospective research study showed that SCI occurred at the spinal levels without an AGOL in 3 wrestlers who sustained SCI during the matches held by the association (cases 19, 24, and 29). These results suggest that the risk of SCI is not so high at the spinal segments with spinal canal stenosis accompanied by an AGOL. This study's results are consistent with those of others. One study showed that players of collision sports tend to develop spinal canal stenosis and osteosclerosis at the cervical spine at earlier ages than ordinary persons, ${ }^{2}$ and another study demonstrated that osseous spinal canal stenosis does not increase the risk of SCI in players of contact sports. ${ }^{13}$ According to the data shown in the latter report, a vertebral body of larger diameter in players decreases the risk of SCI even if they had severe canal stenosis. This result supports our aforementioned

TABLE 2. Summary of the 5 professional wrestlers who had CSI

\begin{tabular}{ccccccccc}
\hline Case No. & Age at CSI (yrs) & AGOL & VA & OPLL & SCS & SCS at Level w/ AGOL & Neck Posture at CSI & CSI \\
\hline 19 & 36 & - & - & C3-4 & + & & Extension & C3-4 SCl \\
\hline 24 & 44 & C4-7 & C4-7 & - & & & Flexion & C3-4 SCl \\
\hline 28 & 36 & - & - & - & & & Flexion & Hangman's fracture \\
\hline 29 & 40 & C4-7 & - & C3-4 & ++ & + & Flexion & C3-4 SCl \\
\hline 30 & 50 & C4-5 & C6-7 & - & + & + & Flexion & C3-4 SCl \\
\hline
\end{tabular}



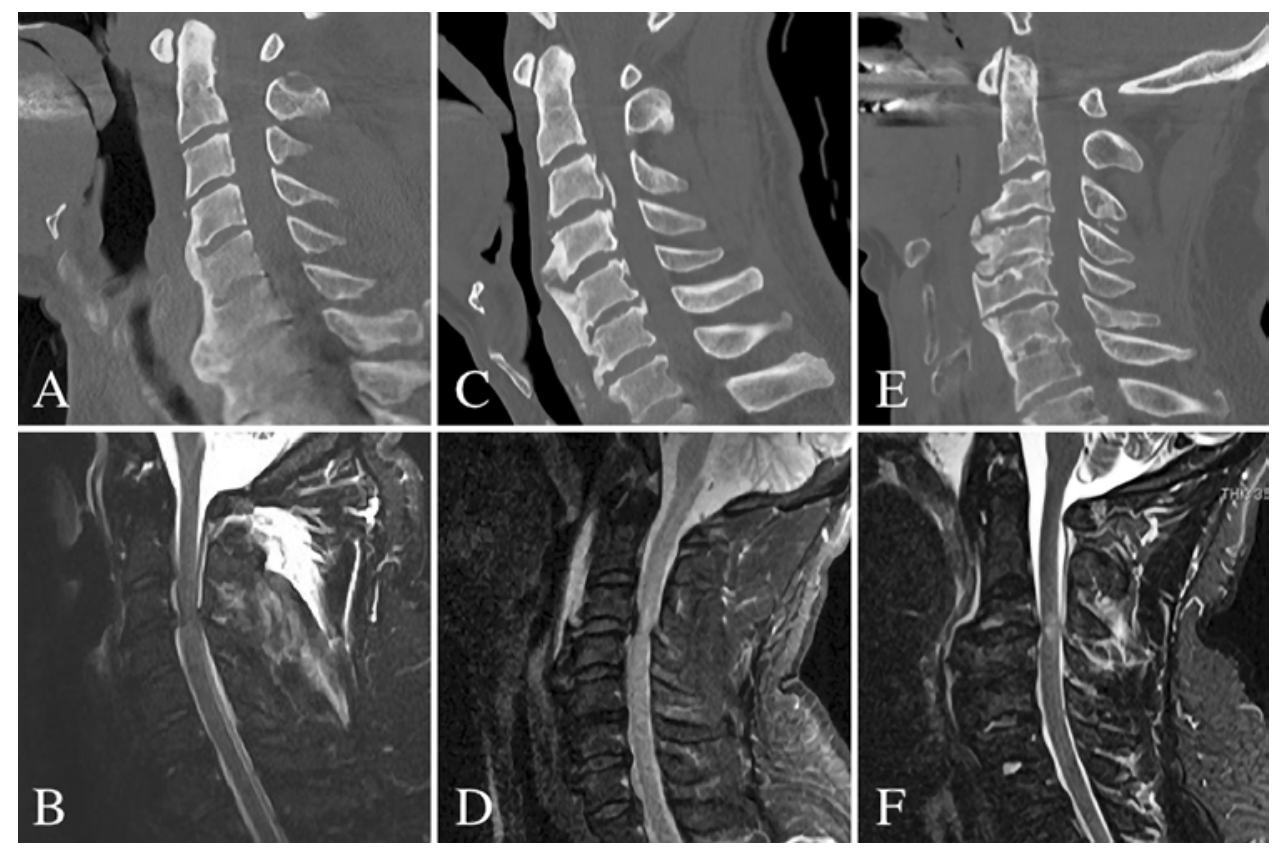

FIG. 2. Imaging studies of the 3 wrestlers (case $24, A$ and B; case $29, C$ and D; and case $30, E$ and F) who sustained SCI during their professional wrestling matches. CT scans (A, C, and E) show a multilevel AGOL below the $\mathrm{C} 4$ level in all 3 wrestlers. MR images (B, D, and F) show intramedullary signal intensity at the C3-4 levels, which suggested that the region of $\mathrm{SCl}$ is adjacent to the AGOL. The CT scan also shows OPLL at the C3-4 levels in 1 wrestler (C), and the MR images show injury of the dorsal soft tissues around the spinous process in 2 wrestlers ( $B$ and $D)$.

consideration that osseous degeneration, such as AGOLs, reinforces the cervical spine against external force. In the present cases, the most frequent pathology of CSI was SCI at the spinal segment adjacent to the multilevel AGOL. As shown in a previous report, ${ }^{10}$ mechanical loading on the neck tended to be focused on the spinal segment adjacent to the location of the AGOL. The multilevel AGOL potentially increased loading on the adjacent spinal segment, and thus increased the risk of SCI.

There were some limitations to our study. First, CSI of the professional wrestlers is a relatively rare incidence in Japan. Second, Japanese professional wrestling associations rarely request resident wrestlers to undergo a medical examination of the cervical spine. Third, longterm follow-up of the professional wrestlers is relatively difficult because they often change their wrestling association affiliation. Therefore, it was challenging to obtain long-term data of a larger number of wrestlers before CSI occurred. We hope that this study leads to further studies investigating the relationship between wrestlers' cervical degeneration and the risk of CSI.

\section{Conclusions}

An AGOL, a giant osteogenic lesion formed at the anterior aspect of the cervical spine, was a peculiar feature of degeneration in Japanese professional wrestlers. Although an AGOL frequently accompanied spinal canal stenosis, it did not increase the risk of SCI. We consider that an AGOL reinforces spinal stability against external forces on the wrestlers' neck during their performance.

\section{Acknowledgments}

We would like to thank Editage for English language editing.

\section{References}

1. Bailey CA, Kukuljan S, Daly RM: Effects of lifetime loading history on cortical bone density and its distribution in middle-aged and older men. Bone 47:673-680, 2010

2. Berge J, Marque B, Vital JM, Sénégas J, Caillé JM: Agerelated changes in the cervical spines of front-line rugby players. Am J Sports Med 27:422-429, 1999

3. Bolam KA, van Uffelen JG, Taaffe DR: The effect of physical exercise on bone density in middle-aged and older men: a systematic review. Osteoporos Int 24:2749-2762, 2013

4. Fujimori T, Watabe T, Iwamoto Y, Hamada S, Iwasaki M, Oda T: Prevalence, concomitance, and distribution of ossification of the spinal ligaments: results of whole spine CT scans in 1500 Japanese patients. Spine (Phila Pa 1976) 41:1668-1676, 2016

5. Hwang JS, Chough CK, Joo WI: Giant anterior cervical osteophyte leading to dysphagia. Korean J Spine 10:200-202, 2013

6. Mizuno J, Nakagawa H, Song J: Symptomatic ossification of the anterior longitudinal ligament with stenosis of the cervical spine: a report of seven cases. J Bone Joint Surg Br 87:1375-1379, 2005

7. Murayama K, Inoue S, Tachibana T, Maruo K, Arizumi F, Tsuji S, et al: Ossified posterior longitudinal ligament with massive ossification of the anterior longitudinal ligament causing dysphagia in a diffuse idiopathic skeletal hyperostosis patient. Medicine (Baltimore) 94:e1295, 2015

8. Nagasawa S, Honda A, Sogo N, Umemura Y: Effects of lowrepetition jump exercise on osteogenic response in rats. $\mathbf{J}$ Bone Miner Metab 26:226-230, 2008

9. Ogul H, Tuncer K, Mutlu V, Gedikli Y, Kantarci M: Giant 
anterior cervical osteophytes leading to dysphagia in a patient with diffuse idiopathic skeletal hyperostosis. Spine J 14:2265, 2014

10. Park MK, Kim KT, Cho DC, Sung JK: Myelopathy associated with instability consequent to resection of ossification of anterior longitudinal ligament in DISH. Eur Spine J [epub ahead of print], 2017

11. Srivastava SK, Bhosale SK, Lohiya TA, Aggarwal RA: Giant cervical osteophyte: an unusual cause of dysphagia. J Clin Diagn Res 10:MD01-MD02, 2016

12. Sundeep M, Hirano Y, Iketani S, Konno A: Surgical management of symptomatic ossified anterior longitudinal ligament: a case report. Surg Neurol Int 8:108, 2017

13. Torg JS, Naranja RJ Jr, Pavlov H, Galinat BJ, Warren R, Stine RA: The relationship of developmental narrowing of the cervical spinal canal to reversible and irreversible injury of the cervical spinal cord in football players. J Bone Joint Surg Am 78:1308-1314, 1996

\section{Disclosures}

The authors report no conflict of interest concerning the materials or methods used in this study or the findings specified in this paper.

\section{Author Contributions}

Conception and design: Sasaki, Asamoto. Acquisition of data: Sasaki, Asamoto, Umegaki. Analysis and interpretation of data: Sasaki, Asamoto. Drafting the article: all authors. Critically revising the article: Sasaki, Asamoto. Reviewed submitted version of manuscript: all authors. Approved the final version of the manuscript on behalf of all authors: Sasaki. Administrative/technical/ material support: Sasaki, Asamoto, Mastumoto. Study supervision: Sasaki, Asamoto.

\section{Supplemental Information}

\section{Previous Presentations}

Portions of this work were presented in abstract form as proceedings at the 52nd Annual Meeting of the Japan Medical Society of Spinal Cord Lesions, Chiba, Japan, on November 16, 2017.

\section{Correspondence}

Manabu Sasaki: Iseikai Hospital, Osaka, Japan. mana-nsu@ umin.net. 